\title{
APPROPRIATE LOCALLY CONVEX DOMAINS FOR DIFFERENTIAL CALCULUS
}

\author{
RICHARD A. GRAFF AND WOLFGANG M. RUESS
}

\begin{abstract}
We make use of Grothendieck's notion of quasinormability to produce a comprehensive class of locally convex spaces within which differential calculus may be developed along the same lines as those employed within the class of Banach spaces and which include the previously known examples of such classes. In addition, we show that there exist Fréchet spaces which do not belong to any possible such class.
\end{abstract}

0. Introduction. In [2], the first named author introduced a theory of differential calculus in locally convex spaces. This theory differs from previous approaches to the subject in that the theory was an attempt to isolate a class of locally convex spaces to which the usual techniques of Banach space differential calculus could be extended, rather than an attempt to develop a theory of differential calculus for all locally convex spaces. Indeed, the original purpose of the theory was to study the maps which smooth nonlinear partial differential operators induce between Sobolev spaces by investigating the differentiability of these mappings with respect to a weaker (nonnormable) topology on the Sobolev spaces.

The class of locally convex spaces thus isolated (the class of $D$-spaces, see Definition 1 below) was shown to include Banach spaces and several types of Schwartz spaces. A natural question to ask is whether there exists an easily-characterized class of $D$-spaces to which both of these classes belong. We answer this question in the affirmative in Theorem 1 below, the proof of which presents a much clearer picture of the nature of the key property of $D$-spaces than the corresponding result [2, Theorem 3.46].

It is immediate from Theorem 1 that every Fréchet quasinormable space is a $D$-space. It is natural to ask at this point whether every Fréchet space is a $D$-space. We answer this question negatively in Theorem 2. Specifically, we show that a Fréchet Montel space is a $D$-space if and only if it is a Schwartz space.

1. Notation and definitions. For $V$ a locally convex space, $V_{b}^{\prime}$ will denote the topological dual space $V^{\prime}$ of $V$, endowed with the (strong dual) topology of uniform convergence on the bounded subsets of $V$. If $U$ is a zero neighbourhood in $V$, then $U^{\circ}$ denotes the polar set of $U$ in $V^{\prime}$, defined by $U^{\circ}=\left\{v^{\prime} \in V|| v^{\prime}(u) \mid \leqslant 1\right.$ for all $u \in U\}$. The space of $r$-linear maps from $V^{r}$ into another locally convex space $W$

Received by the editors July 18, 1981 and, in revised form, March 23, 1982.

1980 Mathematics Subject Classification. Primary 58B10, 58C20, 58C25; Secondary 46A06. 
will be denoted by $L^{r}(V, W)$, and, unless otherwise stated, will be assumed to be endowed with the topology of uniform convergence on the bounded subsets of $V^{r}$.

To maintain consistency with [2], we shall continue to follow the terminology from [9], using the term "compactly generated" when referring to a Hausdorff $k$-space; i.e. a Hausdorff topological space in which subsets are closed if and only if they have closed intersections with each compact subset [5, Chapter 7, p. 230].

Definition 1 [2, Definition 3.21]. A D-space $V$ is a locally convex space with the following property: if $U$ is a neighbourhood of zero in $V, E$ a normed space, $r \in \mathbf{N}$, and $f: U \rightarrow L^{r}(V, E)$ a continuous map, then there exists a neighbourhood $W$ of zero, $W \subset U$, and a continuous seminorm $\nu$ on $V$, such that $f(W) \subset L_{\nu}^{r}(V, E)$ and such that the map $f: W \rightarrow L_{\nu}^{r}(V, E)$ is continuous.

(Here, $L_{\nu}^{r}(V, E)$ denotes the normed linear space $\left\{f \in L^{r}(V, E) \mid \tilde{\nu}(f)<\infty\right\}$, with $\tilde{\nu}$ as norm, where $\tilde{\nu}(f)=\sup \left\{\left\|f\left(x_{1}, \ldots, x_{r}\right)\right\|_{E} \mid \nu\left(x_{i}\right) \leqslant 1,1 \leqslant i \leqslant r\right\}$.)

Definition 2 [2, Definition 3.39]. An exponential space $V$ is a locally convex space such that $V^{r}$ is compactly generated for all $r \in \mathbf{N}$.

REMARK. Let $V$ be a locally convex space, and $Z$ a closed linear subspace of $V$.

(a) If $V$ is an exponential space, then $Z$ and $V / Z$ are exponential spaces.

(b) If $V$ is metrizable, then $V$ is an exponential space.

2. Results. Our results follow by establishing connections between $D$-spaces and the following class of spaces, introduced by A. Grothendieck.

Definition 3 [3, III.1, Definition 4, p. 106]. A locally convex space $V$ is quasinormable, if for every equicontinuous subset $H$ of $V^{\prime}$ there exists a zero neighbourhood $U$ in $V$ such that on $H$ the strong topology (uniform convergence on bounded sets) and the topology of uniform convergence on $U$ coincide.

Equivalently, a locally convex space $V$ is quasinormable, if, for every zero neighbourhood $U$ in $V$, there exists a zero neighbourhood $W$ in $V$ with the following property: for every $\varepsilon>0$, there exists a bounded subset $B_{\varepsilon}$ of $V$ such that $W \subset \varepsilon U+$ $B_{\varepsilon}[3$, III.1, Lemma 6 , p. 107]. (The various formulations of quasinormability and the following example on Schwartz spaces are also to be found in [4, Chapter 3, $\$ 15$, Example 6(c), (d), p. 286].)

EXAMPLES. 1. Every Schwartz space, hence every nuclear space, is quasinormable. More precisely: Schwartz spaces are exactly those quasinormable locally convex spaces whose bounded subsets are precompact [3, III.4, Definition 5, p. 117].

2. Every $D F$ space [3, I.1, Definition 1, p. 63] (see also [6, \$29.3]) and, more generally, every generalized $D F(g D F)$ space in the sense of [8, Definition 2.11], is quasinormable [7, Proposition 3.1]. In particular, given a metrizable locally convex space $Z$, the precompact dual $Z_{p c}^{\prime}$ (uniform convergence on the precompact subsets of $Z$ ), the strong dual $Z_{b}^{\prime}$, and, if $Z$ is complete, also the Mackey dual $Z_{w c}^{\prime}$ (uniform convergence on the weakly compact disks in $Z$ ) are $g D F$, and hence quasinormable.

THEOREM 1. Every quasinormable exponential locally convex space is a $D$-space.

COROLLARY. The following locally convex spaces are D-spaces:

(a) Metrizable quasinormable locally convex spaces, in particular, Fréchet Schwartz spaces. 
(b) Duals of metrizable locally convex spaces, endowed with the topology of uniform convergence on precompact sets. In particular, bw*-spaces ( precompact duals of Banach spaces, [2, Definition 2.19]), and strong duals of Fréchet Montel spaces.

Note that the class of exponential quasinormable locally convex spaces contains normed spaces, all other quasinormable metrizable spaces, and the precompact duals of any of these spaces.

The following proposition, which extends Grothendieck's original characterization of quasinormability, is crucial to the proof of Theorem 1.

Proposition. A locally convex space $V$ is quasinormable if and only if for every normed space $E$ and $r \in \mathbf{N}$ the following property holds: on every equicontinuous subset $H$ of $L^{r}(V, E)$, the topology of uniform convergence on bounded sets coincides with the topology of uniform convergence on a certain zero neighbourhood.

Proof. Sufficiency of the condition being obvious from the definition of quasinormability, only necessity needs to be checked. Assume for the moment that $r=1$ and let $H \subset L(V, E)$ be equicontinuous. Denote by $B$ the norm unit ball of $E$. Then $U=\bigcap_{u \in H} u^{-1}(B)$ is a zero neighbourhood in $V$. Since $V$ is quasinormable, there exists an absolutely convex zero neighbourhood $W$ in $V$ with the following properties:

(i) $W \subset U$;

(ii) For every $\alpha>0$, there exists $M_{\alpha}$ bounded in $V$ such that $W \subset \alpha U+M_{\alpha}$.

Let now $\varepsilon, \rho>0$ be given and let $W_{\rho}^{\varepsilon}=\{u \in L(V, E) \mid u(\rho W) \subset \varepsilon B\}$ be the corresponding zero neighbourhood for the uniform convergence on $W$. According to (ii), there exists $C$ bounded in $V$ such that

(iii) $W \subset\left((2 \rho)^{-1} \varepsilon\right) U+C$.

Let $D=2 \rho C$ and $W_{D}^{\varepsilon}=\{u \in L(V, E) \mid u(D) \subset \varepsilon B\}$, the corresponding zero neighbourhood for the topology of bounded convergence. Then, for $h \in H \cap W_{D}^{\varepsilon}$, we have:

(iv) $h(\rho W) \subset h\left(2^{-1} \varepsilon U+\rho C\right) \subset 2^{-1} \varepsilon B+2^{-1} h(D) \subset \varepsilon B$, i.e.

(v) $H \cap W_{D}^{\varepsilon} \subset W_{\rho}^{\varepsilon}$.

This shows that, on $H$, the topology of bounded convergence is finer than that of uniform convergence on $W$. This completes the proof, for the converse inclusion holds trivially. The proof for $r>1$ is an obvious modification of the above argument.

Proof of Theorem 1. Let $V$ be a quasinormable exponential locally convex space, $U$ an open subset of $V, E$ a normed space, $r \in \mathbf{N}$, and $f: U \rightarrow L^{r}(V, E)$ a continuous map. According to [2, Lemma 3.42, p. 62], the fact that $V$ is exponential implies that the map

$$
g=e v \circ(f \times I): \quad \begin{aligned}
U \times V^{r} & \rightarrow E \\
(u, w) & \mapsto f(u)(w)
\end{aligned}
$$

is continuous. Hence, for a given $x \in U$, the continuity of $g$ at $(x, 0)$ and the fact that $g(x, 0)=0$ together imply that there exists a neighbourhood $U_{x}$ of $x$ and a continuous seminorm $\nu$ on $V$ such that $\|g(y, w)\|_{E} \leqslant 1$ for all $y \in U_{x}$ and for all 
$w=\left(w_{1}, \ldots, w_{r}\right) \in V^{r}$ such that $\nu\left(w_{i}\right) \leqslant 1$. In terms of $f$, this means that $f\left(U_{x}\right)$ is a bounded subset of $L_{\nu}^{r}(V, E)$, and thus that $f\left(U_{x}\right)$ is an equicontinuous subset of $L^{r}(V, E)$. Since $f: U_{x} \rightarrow f\left(U_{x}\right)$ is continuous for the topology of bounded convergence and $f\left(U_{x}\right)$ is equicontinuous, the theorem follows from the above proposition.

We are now going to prove a partial converse to Theorem 1: in order that a Fréchet Montel space (a Fréchet space whose bounded sets are relatively compact) be a $D$-space, it necessarily must be quasinormable, and thus a Schwartz space.

Theorem 2. A Fréchet Montel space is a D-space if and only if it is a Schwartz space.

REMARK. It seems worth noting that the strong dual of any Fréchet Montel space is a $D$-space (Corollary to Theorem 1), whereas the Fréchet Montel spaces themselves are not always Schwartz [6, $\$ 31,5$, p. 433] and hence not $D$-spaces. Interestingly enough, it thus turns out that, among the Fréchet Montel spaces, the (nonlinear) $D$-space property exactly singles out those $F M$-spaces that have the additional (linear) property of being Schwartz.

Proof of Theorem 2. We have to show that, given a zero neighbourhood $U$ in a Fréchet Montel $D$-space $V$, there exists another zero neighbourhood $W$ with the property that, on $U^{\circ} \subset V^{\prime}$, the strong topology and the topology of uniform convergence on $W$ coincide. We first proceed to embed $\left(U^{\circ}, b\right)$ homeomorphically into $V$ and to extend the inverse of this embedding to a continuous map $f$ from $V$ onto $\left(U^{\circ}, b\right)$. An immediate application of the $D$-space property will then complete the proof.

Step 1. Construction of $f$. Since $V$ is a Fréchet Montel space, $\left(U^{\circ}, b\right)$ is a compact separable metric space (see $[6, \S 27,2(5)$, p. 370]). We now use results from infinite-dimensional topology [1]: $\left(U^{\circ}, b\right)$ is homeomorphic to a compact convex subset $C$ of $l^{2}$, which, in turn, is an absolute retract in $l^{2}$ (as usual, $l^{2}$ denotes the Hilbert space of square-summable scalar sequences); and $V$, being an infinite-dimensional separable Fréchet space, is homeomorphic to $l^{2}$. We thus arrive at the following sequence of spaces and mappings: $f: V \approx^{h} l^{2} \stackrel{r}{\rightarrow} C \approx^{g}\left(U^{\circ}, b\right)$, where $h$ denotes a homeomorphism between $V$ and $l^{2}, g$ the homeomorphism between $C$ and $\left(U^{\circ}, b\right)$, and $r$ a continuous retraction from $l^{2}$ onto $C$. If we now let $K=h^{-1}(C)$, then $K$ is a compact subset of $V$ such that $f \mid K$ is a continuous bijection, and thus, by compactness of the spaces involved, a homeomorphism of $K$ onto $\left(U^{\circ}, b\right)$.

Step 2. Let $x \in K$. By the $D$-property of $V$, there exist an open neighbourhood $U_{x}$ of $x$ and a continuous seminorm $\lambda_{x}$ on $V$ such that $f\left(U_{x}\right) \subset L_{\lambda_{x}}(V, \mathbf{R})$ and such that $f \mid U_{x}: U_{x} \rightarrow L_{\lambda_{x}}(V, \mathbf{R})$ is continuous. The compactness of $K$ now assures that there exist $x_{1}, \ldots, x_{n} \in K$ such that $K \subset \cup\left\{U_{x_{i}} \mid i \in\{1, \ldots, n\}\right\}$. Choosing the continuous seminorm $\lambda$ on $V$ to be $\lambda=\sup \left\{\lambda_{x_{1}}, \ldots, \lambda_{x_{n}}\right\}$, we conclude that $\left.f\right|_{K}: K \rightarrow L_{\lambda}(V, \mathbf{R})$ is continuous. Hence, $U^{\circ}=f(K)$ is compact in $L_{\lambda}(V, \mathbf{R})$, i.e. $U^{\circ}$ is compact with respect to the topology of uniform convergence on the zero neighbourhood associated with $\lambda$. Since this topology is at least as fine as the strong dual topology, the two topologies coincide on $U^{\circ}$. This completes the proof. 
Note. Compactness of $U^{\circ}$ for the strong dual topology was crucial to the above demonstration that a Fréchet Montel $D$-space is quasinormable. Thus this technique is not applicable to the non-Montel case, where the bounded sets are not necessarily relatively compact. This follows from the fact that, according to the BanachDieudonné-Theorem [6, $\$ 21,10(1)$, p. 272] and standard results on compactness, a Fréchet space with the property that all equicontinuous subsets of its dual are relatively compact for the strong dual topology, must necessarily be Montel.

\section{REFERENCES}

1. C. Bessaga and A. Pelczynski, Selected topics in infinite-dimensional topology, Monografie Matematyczne, vol. 58, Polish Scientific Publishers, Warsaw, 1975.

2. R. A. Graff, Elements of non-linear functional analysis, Mem. Amer. Math. Soc. 206 (1978).

3. A. Grothendieck, Sur les espaces $(F)$ et ( $D F$ ), Summa Brasil. Mat. 3 (1954), 57-122.

4. J. Horvath, Topological vector spaces and distributions. I, Addison-Wesley, Reading, Mass., 1966.

5. J. L. Kelley, General topology, Van Nostrand, New York-London-Toronto, 1955.

6. G. Köthe, Topological vector spaces. I, Springer-Verlag, Berlin and New York, 1969.

7. W. M. Ruess, On the locally convex structure of strict topologies, Math. Z. 153 (1977), 179-192.

8. Strict topologies and DF spaces, Proc. Paderborn Conf. Functional Anal., 1976, North-Holland Math. Studies, vol. 27, North-Holland, Amsterdam, 1977, pp. 105-118.

9. E. H. Spanier, Algebraic topology, McGraw-Hill, New York, 1966.

The MITRE Corporation, Bedford, MassachusetTs 01730

Fachbereich Mathematik, Universität EsSEN, 43 EsSEn 1, Federal Republic of Germany 